\title{
Oral Prevotella Species and Their Connection to Events of Clinical Relevance in Gastrointestinal and Respiratory Tracts
}

\author{
Eija Könönen* and Ulvi K. Gursoy \\ Institute of Dentistry, University of Turku, Turku, Finland
}

Prevotella is recognized as one of the core anaerobic genera in the oral microbiome. In addition, members of this genus belong to microbial communities of the gastrointestinal and respiratory tracts. Several novel Prevotella species, most of them of oral origin, have been described, but limited knowledge is still available of their clinical relevance. Prevotella melaninogenica is among the anaerobic commensals on oral mucosae from

\section{OPEN ACCESS}

Edited by:

Lucio Souza Gonçalves, Estácio de Sá University, Brazil

Reviewed by: Latifa Bousarghin, University of Rennes 1, France Zhenjiang (Zech) Xu, Nanchang University, China Roland Arnold,

The University of North Carolina at Chapel Hill, United States

*Correspondence: Eija Könönen eija.kononen@utu.fi

Specialty section:

This article was submitted to Microbial Symbioses, a section of the journal

Frontiers in Microbiology

Received: 20 October 2021 Accepted: 14 December 2021 Published: 06 January 2022

Citation: Könönen E and Gursoy UK (2022) Oral Prevotella Species and Their Connection to Events of Clinical Relevance in Gastrointestinal and Respiratory Tracts.

Front. Microbiol. 12:798763. doi: 10.3389/fmicb.2021.798763 early months of life onward, and other early colonizing Prevotella species in the oral cavity include Prevotella nigrescens and Prevotella pallens. Oral Prevotella species get constant access to the gastrointestinal tract via saliva swallowing and to lower airways via microaspiration. At these extra-oral sites, they play a role as commensals but also as potentially harmful agents on mucosal surfaces. The aim of this narrative review is to give an updated overview on the involvement of oral Prevotella species in gastrointestinal and respiratory health and disease.

Keywords: anaerobic bacteria, commensalism, dysbiosis, inflammation, microbiology, Prevotella, systemic disease, taxonomy

\section{INTRODUCTION}

Anaerobic bacteria constitute a significant part of oral microbial communities. In the oral cavity, Bacteroidetes is one of the major phyla and Prevotella its largest genus (Dewhirst et al., 2010). This genus, which has expanded significantly during the past decades, consists of gram-negative, strictly anaerobic, mainly short rod-shaped bacteria. In healthy adults, detection rates of Prevotella organisms are high in saliva and dental plaque (Keijser et al., 2008; Xu et al., 2015). In saliva, richness of the diversity within this genus is especially high (Keijser et al., 2008). At the species level, however, the majority of data currently available deals with Prevotella intermedia and/or Prevotella nigrescens due to their clinical relevance in oral pathologies but also ignorance of commensals. A study looking for intraoral distribution of bacterial species in 225 systemically healthy individuals showed Prevotella melaninogenica in high proportions in saliva and at the dorsum and lateral sites of the tongue (Mager et al., 2003).

In the context of this review, human habitats exposed to oral bacteria to be colonized outside the oral cavity are presented in Figure 1. There are obvious differences in the microbial communities between body habitats and between individual metabolic niches (Costello et al., 2009; Segata et al., 2012). At the genus level, Prevotella is frequent and widespread all over on the surfaces of the human body; however, a species-level identification methodology is necessary for revealing whether 


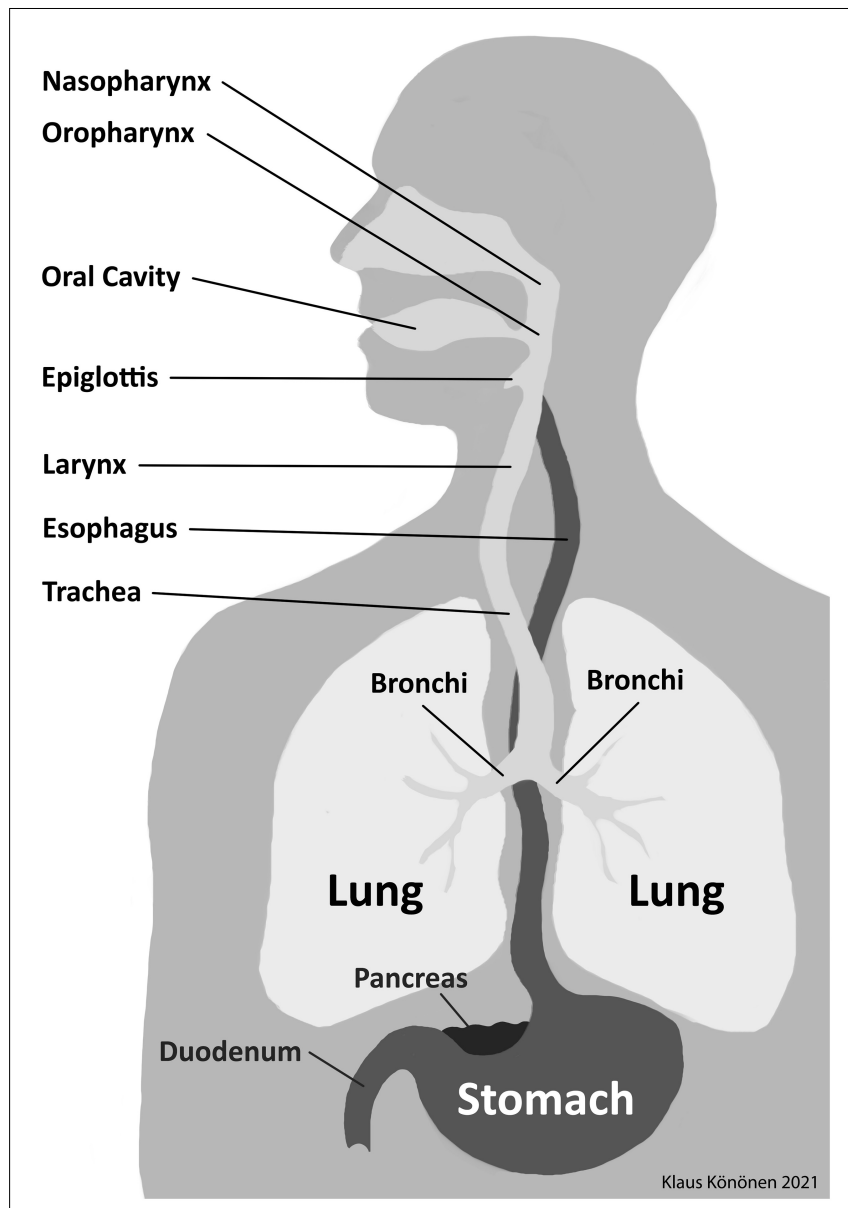

FIGURE 1 | The anatomy and potential habitats in the aerodigestive tract in humans.

same species colonize throughout the gastrointestinal tract and whether their relative abundance varies among the habitats as well as inter-individually (Segata et al., 2012; Schmidt et al., 2019). Prevotella strains present in saliva are of particular interest. Due to constant saliva swallowing of approximately $1.5 \mathrm{~L}$ daily (Humphrey and Williamson, 2001), this oral fluid is the most plausible vehicle for oral microorganisms and their biologically active components to be translocated to other parts of the digestive tract (Schmidt et al., 2019). Oral bacteria surviving to pass acidic circumstances of the stomach get access to the small intestine and colon, where they can interfere with intestinal bacteria (Schmidt et al., 2019; Kitamoto et al., 2020). At the genus level, Prevotella is a common colonizer of these distant habitats. However, at the species level, distinct bacterial populations occupy the oral and intestinal microenvironments. Also immune cells involved in chronic inflammatory processes in the mouth end up via saliva to the gut, sometimes resulting in pathological consequences (Byrd and Gulati, 2021).

In the respiratory tract, potential routes for bacterial translocation from the oral cavity include (micro)aspiration, in particular, and hematogenous spread. Indeed, recent studies have shaken our views on the oral source of bacteria for the composition of microbial communities of the lower respiratory tract (Bassis et al., 2015; Dickson et al., 2016). Currently, Prevotella is considered one of the major genera colonizing mucosal surfaces of the aerodigestive tract, including the lungs, which were long seen as a sterile site in the human body.

In this narrative review, the purpose is to give an updated overview on the presence of oral Prevotella species as members of the gastrointestinal and respiratory microbiota and on their involvement in diseases at these sites above the waistline.

\section{TAXONOMICAL OVERVIEW OF ORAL PREVOTELLA}

In 1990, the genus Prevotella was described by reclassifying a group of moderately saccharolytic and bile-susceptible Bacteroides species as members of this novel genus (Shah and Collins, 1990). The name Prevotella came after A. R. Prévot, who was a French microbiologist with pioneering expertise in anaerobic bacteriology. Most of these reclassified Prevotella organisms were recovered from the oral cavity of humans, $P$. melaninogenica being the type species of the genus. At that time, pigment production of colonies on blood agar was seen as an important feature to divide the organisms into pigmented and non-pigmented Prevotella species (Jousimies-Somer et al., 2002). In addition to $P$. melaninogenica, the pigmented group included Prevotella loescheii, Prevotella denticola (part of strains), P. intermedia, and Prevotella corporis, their color intensity varying from light brown to black. Some years later, the pigmented group within Prevotella was expanded by two phylogenetically closely related species, $P$. nigrescens (Shah and Gharbia, 1992) and Prevotella pallens (Könönen et al., 1998). During the 1990s, there was a notable research interest in blackpigmented gram-negative anaerobes, including Porphyromonas and Prevotella species, due to their link to various pathologies in humans (Finegold et al., 1993).

After the creation of the genus Prevotella and reclassification of moderately saccharolytic Bacteroides as Prevotella species by Shah and Collins (1990), there has been a great expansion of the genus with novel species, most of them of oral origin. Table 1 presents the validly published Prevotella species that have been primarily isolated from the oral cavity: 12 species formerly classified as Bacteroides and 18 novel Prevotella species described after 1990. On the other hand, a couple of reclassifications were made; while the former Mitsuokella dentalis is now Prevotella dentalis (Willems and Collins, 1995), Prevotella tannerae was removed to a novel, closely related genus Alloprevotella, also including a novel oral species, Alloprevotella rava (Downes et al., 2013). Two species with a distant phylogeny, Prevotella heparinolytica and Prevotella zoogleoformans, remain "on the waiting list" to be removed from the genus Prevotella (Dewhirst et al., 2010).

In addition to the above-mentioned species principally isolated from the oral cavity, several novel Prevotella species have been described based on a few strains, or even a single strain, from various clinical specimens. Origins of these strains were as follows: two Prevotella amnii strains from amniotic fluid 
TABLE 1 | Validly published Prevotella and Alloprevotella species with the primary isolation from the human oral cavity.

\begin{tabular}{|c|c|c|c|c|}
\hline Year/Reference & $\begin{array}{l}\text { Former Bacteroides sp. reclassified } \\
\text { as Prevotella sp. }\end{array}$ & Year/References & $\begin{array}{l}\text { Novel Prevotella and } \\
\text { Alloprevotella spp. }\end{array}$ & Comments \\
\hline \multirow[t]{15}{*}{ Shah and Collins, 1990} & $\begin{array}{l}\text { P. melaninogenica } \\
\text { (type species) }\end{array}$ & Shah and Gharbia, 1992 & P. nigrescens & \\
\hline & P. buccae & Moore et al., 1994 & $\begin{array}{l}\text { P. enoeca } \\
\text { P. tannerae }\end{array}$ & \\
\hline & P. buccalis & Willems and Collins, 1995 & P. dentalis & Mitsuokella dentalis \\
\hline & P. denticola & Könönen et al., 1998 & P. pallens & \\
\hline & P. heparinolytica & Sakamoto et al., 2004 & $\begin{array}{l}\text { P. salivae } \\
\text { P. shahii }\end{array}$ & \\
\hline & P. intermedia & Sakamoto et al., 2005a & P. multiformis & \\
\hline & P. loescheii & Sakamoto et al., 2005b & P. multisaccharivorax & \\
\hline & P. oralis & Downes et al., 2005 & $\begin{array}{l}\text { P. baroniae } \\
\text { P. marshii }\end{array}$ & \\
\hline & P. oris & Downes et al., 2007 & P. maculosa & \\
\hline & P. oulorum & Downes et al., 2008 & P. histicola & \\
\hline & P. veroralis & Downes et al., 2009 & P. micans & \\
\hline & P. zoogleoformans & Sakamoto et al., 2010 & P. aurantiaca & \\
\hline & & Downes et al., 2010 & P. saccharolytica & \\
\hline & & Downes and Wade, 2011 & $\begin{array}{l}\text { P. fusca } \\
\text { P. scopos }\end{array}$ & \\
\hline & & Downes et al., 2013 & $\begin{array}{c}\text { A. rava } \\
\text { A. tannerae }\end{array}$ & $\begin{array}{l}\text { Alloprevotella gen. nov., } \\
\text { reclassification of } P \text {. tannerae }\end{array}$ \\
\hline
\end{tabular}

(Lawson et al., 2008), eight Prevotella bergensis strains from skin and soft tissue infections (Downes et al., 2006), one Prevotella brunnea strain from a wound at foot (Buhl et al., 2019), one Prevotella colorans strain from a wound (Buhl et al., 2016), three Prevotella jejuni strains from the jejunum of a celiac child (Hedberg et al., 2013), three Prevotella nanceiensis strains from bronchial fluid, lung abscess, or blood (Alauzet et al., 2007), one Prevotella pleuritidis strain from pleural fluid (Sakamoto et al., 2007), one Prevotella timonensis strain from breast abscess (Glazunova et al., 2007), and one Prevotella vespertina strain from an abscess locating at the upper respiratory tract (Buhl and Marschal, 2020). With this limited information given, the knowledge of their preferred habitat remains open.

Three former Bacteroides species, reclassified as Prevotella bivia, Prevotella corporis, and Prevotella disiens (Shah and Collins, 1990) come mainly from the human urogenital tract but there are also occasional recoveries from the mouth. In the current literature, several novel Prevotella species from stool specimens have been described as colonizers of the colon. Of those, Prevotella copri has drawn remarkable attention due to its potential beneficial effects on human well-being (Tett et al., 2021), although its connections to chronic inflammatory conditions of the gut have been recognized, too (Ley, 2016).

Besides biochemical and physiological testing, methods for Prevotella classification have traditionally included DNA-DNA hybridization, measuring of $\mathrm{G}+\mathrm{C}$ content, and multilocus enzyme electrophoresis, however, classification gradually changes toward genomic methods. Full-sequencing of the $16 \mathrm{~S}$ rRNA gene is a routine, but also comparison to whole-genome sequence databases is available and has been used in recent Prevotella descriptions (Buhl et al., 2016, 2019; Buhl and Marschal, 2020). Tett et al. (2021) recently described genomic characteristics of 25 named human Prevotella species, among those 17 species with genomes of multiple oral isolates in the analysis. Varying genome lengths between 2.37-4.26 Mb and $\mathrm{G}+\mathrm{C}$ contents between 36.4$56.1 \%$ as well as the high number of core genes speak for the wide diversity within this genus.

\section{PREVOTELLA IN BACTERIAL COMMUNITIES OF THE DIGESTIVE TRACT}

\section{Prevotella as Members of the Core Microbiota in the Gastrointestinal Tract}

Concerning the alimentary tract from the oropharynx downward above the waistline, it was for long considered of being without a resident microbiota until advanced molecular methods allowed to challenging these earlier beliefs, which were based on negative cultures by routine microbiology methods from the esophagus and stomach (Pei et al., 2004; Bik et al., 2006). Pei et al. (2004) tested their hypotheses on the presence of indigenous bacteria on esophageal mucosa and on their fastidious nature, i.e., mainly being uncultivable. Biopsy specimens from the distal esophagus of four healthy individuals were examined with $16 \mathrm{~S}$ rDNA sequencing-based techniques, and the results showed Bacteroidetes as the second most common phylum and Prevotella as the second most common genus after Streptococcus. $P$. pallens was among the 14 bacterial taxa found in all individuals, while many other named Prevotella species were also recovered: Prevotella veroralis from three individuals, $P$. intermedia and $P$. melaninogenica from two individuals, and $P$. denticola, 
P. nigrescens, and Prevotella oris each from one individual. In addition, several not-yet-named Prevotella clones were among the findings (Pei et al., 2004). To increase the understanding of the esophageal microbiome and its function in the host, Deshpande et al. (2018) studied the esophageal microbiome of over 100 individuals, with special emphasis on age, gender, proton pump inhibitor use, host genetics, and development of esophageal disease. They demonstrated that the esophageal microbiome clusters into functionally distinct bacterial community types. Cluster 3 was dominated by $P$. melaninogenica and $P$. pallens, and cluster 2 by mitis group streptococci, while cluster 1 was an intermediate type with respect to abundances of Streptococcus and Prevotella, indicating their ratio of being a significant factor in defining esophageal community types (Deshpande et al., 2018). It was shown that various, distinct pathways are increased in each community type. Age was observed to affect relative abundances within these two major genera; age positively correlated with Streptococcus parasanguinis (but not with mitis group streptococci), whereas age had an inverse correlation with $P$. melaninogenica (but not with $P$. pallens). Interestingly, there was a co-exclusion relationship between mitis group streptococci and Prevotella species across all esophageal disease stages (Deshpande et al., 2018).

Bik et al. (2006) collected gastric biopsy specimens from 23 adults, suffering from symptomatic upper gastrointestinal disease and being positive or negative for Helicobacter pylori. Advanced sensitive methods were used to reveal the bacterial composition of the gastric microbiota and the impact of H. pylori on its composition. There was a much larger bacterial diversity in gastric biopsies than was expected, but the sequence collection was also different from those of the mouth and esophagus presented in other studies. Interestingly, especially Bacteroidetes phylotypes were often absent from H. pylori-positive individuals (Bik et al., 2006). However, several recognized oral Prevotella species (and several Prevotella clones) were detected in individuals who were $H$. pylori-negative by conventional methods. Among the findings were, in descending order in their abundance, P. melaninogenica and P. pallens, in particular, and few $P$. oris, $P$. intermedia, $P$. nigrescens, Prevotella oralis, Prevotella oulorum, and $P$. denticola. It was emphasized that bacterial DNA may not indicate the presence of resident bacteria but, instead, could reflect the presence of bacterial cell remnants or transient flow of these bacteria in the specimens (Bik et al., 2006). Recently, it was confirmed that H. pylori, if present, dominates the bacterial community composition of the stomach, lowering the diversity and evenness of phylotypes (Schulz et al., 2018). Similarly to the study by Bik et al. (2006), two genera, Streptococcus and Prevotella, proved to dominate in bacterial communities of the upper gastrointestinal tract in $H$. pylori-negative individuals. By analyzing saliva, and stomach and duodenal aspirates and biopsies from a cohort of 24 individuals with chronic gastritis by high-throughput sequencing, Schulz et al. (2018) identified the oral cavity (saliva) of being the main source of active bacteria for the gastric microbiota, indicating a continuous migration of oral bacteria through the upper gastrointestinal tract. In individuals without $H$. pylori, no difference was seen in relative abundance of Prevotella and Alloprevotella between saliva and stomach aspirates, while reduced abundance for both genera was detected between the stomach and duodenum. In general, no significant difference at the genus level was observed in oral communities between individuals with or without $H$. pylori, but three phylotypes, among those P. oris, had significantly higher abundance in individuals without H. pylori (Schulz et al., 2018). Each individual harbored own specific bacterial communities throughout the upper gastrointestinal tract.

Saliva has been suggested as a key driver for the composition of bacterial communities in various habitats of the upper digestive tract, and as another key driver, a shared epithelial lining of their mucosae (Segata et al., 2012). Vasapolli et al. (2019) looked thoroughly for bacterial community changes in 21 healthy individuals throughout their gastrointestinal tract, including a wide selection of specimens: saliva, stomach (antrum and corpus), duodenum, terminal ileum, ascending and descending colon, and feces. They demonstrated that bacterial communities detected in samples from saliva, stomach, and duodenum formed 1 cluster and those from the lower gastrointestinal tract another cluster, while fecal communities differed from mucosaassociated findings of the gut (Vasapolli et al., 2019). The highest phylotype richness, including the genus Prevotella, was found in saliva, staying rather steady across the upper body sites, whereas in samples from lower parts of the body, a significantly reduced phylotype richness was observed. Among the phylum Bacteroidetes, abundance of Prevotella organisms present in saliva gradually decreased downward up to the duodenum, the decrease being drastic thereafter, whereas an opposite occurred to abundance of Bacteroides organisms (Vasapolli et al., 2019). In line were the results of a thorough culture-based study, performed in a homologous group of beagle dogs (Mentula et al., 2005), showing clear differences between jejunal and fecal samples and where a unique bacterial composition for each dog was found in small-intestinal fluid with a few species only at a time and fluctuating counts. Smoking seems to be a lifestyle factor affecting the mucosa-associated microbiota of the duodenum; in current smokers, a significantly reduced abundance of the phylum Bacteroidetes and the genus Prevotella was observed, and this reduction was only partially restored after quitting of smoking (Shanahan et al., 2018). P. nanceiensis was suggested to be a discriminatory species for previous smokers.

A few Prevotella species isolated from feces are considered having their habitat in the intestine. According to the current knowledge, especially $P$. copri is both a prevalent and abundant organism in the gut [see an excellent review by Tett et al. (2021)]. Interestingly, lifestyle factors, diet in particular, have a significant impact on its abundance. In non-Westernized populations with diets rich of carbohydrates and fibers, the P. copri complex is a common inhabitant of the gut, while different bacterial taxa dominate in populations with Western-type diets. On one hand, the $P$. copri complex seems to possess beneficial metabolic effects on human health but, on the other hand, potential detrimental effects may also exist (Ley, 2016; Tett et al., 2021). The impact of diet on oral Prevotella species is not known. 


\section{Prevotella Involvement in Gastrointestinal Diseases}

In the human esophagus, gastroesophageal reflux disease is a rather common pathological condition, where the relaxation of the sphincter muscle at the lower esophagus allows acidic stomach contents frequently flow back into the esophagus and further into the pharyn $x^{1}$. This constant leakage irritates the epithelial surface, causing reflux symptoms or esophagitis, which may lead to Barrett's esophagus with a disturbed epithelium structure. The potential role of changes in the esophageal and/or salivary microbiota has been studied and, in this context, the genus Prevotella is of interest (Liu et al., 2013; Kawar et al., 2021). Bacterial communities of the distal esophagus were examined at the phylum and genus levels in biopsy samples collected from Japanese patients with either normal esophagus, reflux esophagitis, or Barrett's esophagus (Liu et al., 2013). The study revealed Bacteroidetes among the major phyla, and the proportions of Prevotella clones in the samples were 3, 5, and $12 \%$, respectively. Of the six patients in each group, the number of Prevotella-positive patients was three, four, and six, respectively. A distinct bacterial composition seen between the groups was assumed to indicate an association with an esophageal health status and disease type (Liu et al., 2013). Recently, Kawar et al. (2021) compared the bacterial composition of salivary samples collected from reflux patients treated with proton pump inhibitors to that from non-medicated reflux patients and healthy controls. Abundant taxa present in the samples from the latter two groups differed considerably, including reduced abundances of $P$. melaninogenica and $P$. pallens in saliva of reflux patients. It was assumed that a lowered $\mathrm{pH}$ in the oral cavity of nonmedicated patients explains this difference, since no significant difference was found between saliva of medicated reflux patients and healthy controls (Kawar et al., 2021).

Bacterial compositions of the stomach and duodenum were examined in 98 Korean patients with symptomatic gastritis (Han et al., 2019). It was demonstrated that the gastric and duodenal microbiota differ from each other. Symptom scores only weakly correlated with abundance of gastric $H$. pylori but, instead, correlated more strongly with the duodenal microbiota. An interesting gender-related finding was the higher relative abundance of $P$. pallens in the stomach of women than in that of men. There was a negative correlation with relative abundance of $P$. pallens and the severity of symptoms in the stomach. Positive correlations were found with $P$. nanceiensis and A. rava in the duodenum. According to the authors, other factors than H. pylori need to be taken into account in symptomatic gastritis (Han et al., 2019).

A novel Prevotella species was detected for the first time in a biopsy taken from the jejunum of a child with celiac disease and was named as P. jejuni due to its localization in this part of the small intestine (Hedberg et al., 2013). However, this species seems to be a common inhabitant of the oral cavity and especially in saliva, similarly to its close relative, P. melaninogenica (own unpublished data). Alterations in the salivary microbiota and

\footnotetext{
${ }^{1}$ https://www.hopkinsmedicine.org/gastroenterology_hepatology/_pdfs/ esophagus_stomach/gastroesophageal_reflux_disease.pdf
}

metabolome in celiac children, who had been under glutein-free diet at least for 2 years, were examined and compared to those of healthy control children (Francavilla et al., 2014). The number of total cultivable anaerobes differed between the groups, with reduced amounts found in children with celiac disease. Members of the phylum Bacteroidetes, among those $P$. nanceiensis, dominated in saliva, with increased abundance in celiac children. It was concluded that the diet change of 2 years may not be enough to restore the salivary microbiota (Francavilla et al., 2014).

Although the relation of the oral microbiota to inflammatory bowel diseases (IBD) is still controversial, there is evidence on their connection to dysbiotic bacterial communities in saliva (Said et al., 2014; Xun et al., 2018; Qi et al., 2021). Dysbiosis with an increased relative abundance of Bacteroidetes, in turn, causes an elevated inflammatory response, which is also seen in saliva as increased levels of cytokines like interleukin-1 $\beta$ (Said et al., 2014). No alterations were observed in the richness and diversity of bacterial communities between saliva from IBD patients and healthy controls, whereas the bacterial composition in saliva varied (Said et al., 2014; Qi et al., 2021). While abundance of Streptococcus decreased, that of Prevotella (besides Veillonella) and P. melaninogenica significantly increased in both Crohn's disease and ulcerative colitis patients. The genus Prevotella was suggested as a potential indicator related to Crohn's disease (Qi et al., 2021). Moreover, the dysbiotic salivary composition contributed to aggravated immune disorders in IBD patients.

\section{Prevotella in Cancers of the Digestive Tract Above the Waistline}

In the esophagus, cancer types differ depending on the geographical location; in the East, squamous cell carcinoma (SCC) dominates, whereas adenocarcinoma is more common in Western countries (Sung et al., 2021). In the first study to examine bacterial infection as a background factor for esophageal SCC and to identify bacteria that could predict the cancer prognosis, the genus Prevotella came up as a potential prognostic indicator for this cancer type (Liu et al., 2018). Patients with lymph node metastasis had higher abundance of Prevotella and Treponema than patients without metastatic findings, while increased abundance of Prevotella combined with Streptococcus affected survival rates, predicting poor prognosis. Alterations of the esophageal microbiota have also been studied in connection to Barrett's esophagus and esophageal adenocarcinoma (Lopetuso et al., 2020). The former condition with changes in the epithelial structure with acid stress and inflammation is considered to expose to metaplastic mucosa. Again, abundance of Prevotella was significantly increased, however, species-level shifts showed decreased proportions of $P$. melaninogenica in samples from Barrett's esophageal mucosa with or without cancer but increased proportions of unclassified Prevotella. P. histicola was common on mucosae of Barrett's esophagus, while P. nigrescens proportions on metaplastic mucosa were slightly increased (Lopetuso et al., 2020). The authors reported a coexclusion association between Streptococcus and Prevotella; a significant reduction in relative abundance of Streptococcus and 
corresponding increase in abundance of Prevotella were observed on mucosae of both Barrett's esophagus and adenocarcinoma.

A decade ago, bacterial communities of the stomach in gastric cancer patients were described for the first time by molecular techniques and compared to those in dyspeptic patients with normal mucosa as controls (Dicksved et al., 2009). The second most dominant phylum proved to be Bacteroidetes, composing mainly of Prevotella taxa; among known species, Prevotella multiformis, $P$. nigrescens, $P$. oris, and A. tannerae were recognized in gastric cancer patients. It can be speculated whether changed conditions due to an increased use of acid-reducing drugs or neoplastic mucosa would allow the colonization of atypical bacteria in the stomach and progression of cancer (Dicksved et al., 2009; Coker et al., 2018). Coker et al. (2018) investigated mucosal biopsy specimens collected from superficial gastritis, atrophic gastritis, intestinal metaplasia, and gastric cancer patients, and detected highest abundance of several oral bacteria, among those $P$. intermedia and $P$. oris, in gastric cancer samples. It was emphasized, however, that it remains to be elucidated in targeted studies whether bacteria enriched are passengers or drivers of carcinogenesis (Coker et al., 2018).

Poor oral hygiene is suggested as a moderate risk factor for pancreatic cancer (Huang et al., 2016). This may be connected to increased amounts of microbial biofilms on oral surfaces and activation of host inflammatory response. In a recent study, a large variety of samples from the gastrointestinal tract, including oral (saliva and swabs from tongue, buccal mucosa, and gingiva), upper intestinal (duodenum tissue and swabs from jejunum and bile duct), and pancreatic (tumor or normal tissue and pancreatic duct) samples were examined to clarify the microbiota in patients with pancreatic cancer or other diseases of the pancreas or the foregut (Chung et al., 2021). Streptococcus, Veillonella, and Prevotella were the most shared genera between oral and gut or pancreatic samples, and $P$. veroralis among the most frequently shared species. Amplicon Sequence Variants had some overlaps between the close sites within the mouth and within the pancreas. In co-abundance analyses, distinct strain-level cluster patterns were observed among microbial findings in buccal swabs, saliva, duodenal tissue, jejunal swabs, and pancreatic tumor tissue. In the latter sample site, $P$. nigrescens was found among dominating species in one cluster (Chung et al., 2021).

\section{PREVOTELLA IN BACTERIAL COMMUNITIES OF THE RESPIRATORY TRACT}

\section{Prevotella as Members of the Core Microbiota in the Respiratory Tract}

A gradual maturation of the early microbiota of the lower respiratory tract occurs within less than 2 months after birth in full-term infants (Pattaroni et al., 2018). Three distinct colonization patterns were recognized in tracheal aspirates and were explained by distinct microenvironments in preterm and term infants. Of those, a mixed microbial profile consisted of a balanced composition of six genera, including
Streptococcus and Neisseria as keystone genera, and anaerobic Prevotella, Veillonella, Porphyromonas, and Fusobacterium. This combination stayed stable across the first year of life (Pattaroni et al., 2018). Interestingly, this is a typical bacterial composition for the early microbiota established in the mouth during the first year of life (Könönen et al., 1999; Könönen, 2005). It also resembles the composition of the lung microbiota of adults during health (Bassis et al., 2015).

Similar interacting consortia as seen in the oral cavity (but not in the nasal cavity) can be observed in the lower respiratory tract, even though relative abundance and diversity richness are lower in the lungs (Bassis et al., 2015). Indeed, the oropharynx is considered the principal origin for the lung bacteriome during health (Bassis et al., 2015; Dickson et al., 2016). At the genus level, Prevotella is consistently among the core bacterial communities of the respiratory tract (Bassis et al., 2015; Einarsson et al., 2019). However, methodologies assessing the bacterial taxa at the species level, and even at the strain level, are necessary for discovering their source and role as beneficial, harmless or potentially harmful members of the bacterial communities at a specific body site. Noteworthy is that the Prevotella genus accommodates a high number of species with distinct clinical significance.

Valuable, detailed species-level oropharyngeal data assessed by in-depth sequencing are available from a study where tonsillar crypts in 2- to 4-year-old children and young adults were examined during recurrent tonsillitis and were compared to tonsillar crypts in children with tonsillar hyperplasia but without inflammation and those in healthy adult controls (Jensen et al., 2013). Streptococcus and Prevotella were found in all 20 samples from children and high Prevotella abundance was observed. Recurrent tonsillitis associated with a shift in the bacterial composition, especially with increased $P$. melaninogenica/P. histicola in adults. Typical oral Prevotella species, including Prevotella buccae, P. dentalis, P. denticola, Prevotella fusca, Prevotella micans, $P$. oralis, $P$. oris, $P$. pallens, Prevotella salivae, and $P$. veroralis, were more abundant in adults but Prevotella saccharolytica in children. $P$. intermedia was absent, except for a healthy adult with a high quantity. The recoveries of $P$. nanceiensis and $P$. pleuritidis from the oropharynx give support for their oral habitat. These study results indicate that a core microbiome, with a few significant genera, is present in tonsillar crypts regardless of individuals' age and health status (Jensen et al., 2013).

Recently, a potential link between the microbial community composition and lung homeostasis was examined by analyzing bronchoalveolar lavage samples from a longitudinally followed post-transplant study population (Das et al., 2021). Although the lung microbiota proved to be highly variable, there were a few bacterial taxa with high prevalence and/or abundance, and the majority of them were either obligate or facultative anaerobes. Among the most prevalent species were P. melaninogenica, Veillonella atypica, Veillonella dispar, Streptococcus mitis, and Granulicatella adiacens (all typical recoveries from the oral cavity). The microbiota was categorized into four distinct compositional states (pneumotypes) where the balanced pneumotype represented a diverse bacterial community, resembling that in the oropharynx and including 
P. melaninogenica, which occurred in $97.4 \%$ of the samples (Das et al., 2021). In addition, this pneumotype had a high immunemodulatory activity and preserved lung stability.

Although oral bacteria get access to proximal airways via microaspiration, their growth at high densities is prevented by the continuous mucociliary clearance (Bassis et al., 2015). A recent study using a mouse model (Wu et al., 2021) demonstrated that the episodic aspiration of oral commensals, such as P. melaninogenica, Veillonella parvula, and S. mitis, leads to dysbiosis and low-dose inflammation in the lower airways; the consequence is a reduced susceptibility to pathogenic Streptococcus pneumoniae via activation of pulmonary Th17 cells. The shift in the human lung microbiome from the phylum Bacteroidetes in health to Gammaproteobacteria in disease indicates that Prevotella-activated Th17 response is an essential part of the pathogen recognition and suppression system of a healthy lung environment (Huffnagle et al., 2017). Anaerobic bacteria, especially Prevotella, are frequent recoveries from clinical respiratory specimens; however, understanding of their contribution to lung diseases is not clear yet.

\section{Prevotella Involvement in Acute Diseases of the Respiratory Tract}

Along with high research interest targeted to the COVID-19 pandemic, an increasing number of reports on the potential involvement of oral bacteria in the disease persistence and treatment outcome are available in the current literature. Interestingly, also Prevotella organisms, being analyzed from salivary, oropharyngeal, and bronchoalveolar lavage samples examined for SARS-CoV-2, have come up recently in this context. The microbiome and SARS-CoV-2 viral loads in saliva were compared between hospitalized COVID-19 and control patients (Miller et al., 2021). Although no significant difference in their bacterial compositions was found, the abundance of some taxa associated with the viral load in saliva; here, of special interest is enriched $P$. pallens but reduced $P$. denticola and $P$. oris in saliva of COVID-19 patients (Miller et al., 2021). Xiong et al. (2021) examined the difference in the microbial composition between SARS-CoV-2-positive and -negative pharyngeal swab samples collected from symptomatic patients with cough and fever. A significantly reduced species richness was seen in COVID19 samples. The top-3 genera enriched were Streptococcus, Prevotella, and Campylobacter. Changes in abundance were seen also for several Prevotella species, such as $P$. denticola, P. oris, $P$. jejuni, $P$. intermedia, P. melaninogenica, P. fusca, and $P$. scopos, which were among the 37 species distinctive for the healthy and diseased individuals, and most of them separated the symptomatic COVID-19 and non-COVID groups from each other (Xiong et al., 2021). A dysbiotic oropharyngeal microbiota with gram-negative commensals, considered pathobionts due to their lipopolysaccharide production, has been connected to a so-called long-COVID disease (Haran et al., 2021). Among 164 patients with various types of symptoms, increased abundances of several Prevotella species were among the top predicting taxa from swabs of the posterior oropharynx: P. denticola, P. nigrescens, $P$. histicola, and $P$. oulorum in the patient group with ongoing symptoms, and $P$. denticola, $P$. melaninogenica, $P$. jejuni, and $P$. nigrescens in the determined long-COVID group (Haran et al., 2021). Sulaiman et al. (2021) examined a hospitalized cohort of 589 critically ill COVID-19 patients, characterizing their lung microbiome from bronchoalveolar lavage samples. Especially interesting were the findings of two oral commensals, $P$. oris and Mycoplasma salivarium, among the most dominant, functionally active microbial taxa. While $M$. salivarium was enriched in the deceased and $>28$-day mechanically ventilated groups, $P$. oris was enriched in the $\leq 28$ day group. It was suggested that dissimilar microbial pressures related to host factors could explain the difference between the groups (Sulaiman et al., 2021). These studies indicate that the microbiome of the host plays a role in COVID-19 outcome.

Oral bacteria present in saliva can promote aspiration pneumonia via colonizing on mucosal surfaces, affecting immune response of epithelial cells, and producing proinflammatory cytokines and degradative enzymes, but dispersal via hematogenous route is also an option (Scannapieco, 2021). Older age and supine position as well as poor oral hygiene increase the risk for aspiration of bacteria from the oral cavity to lower parts of the respiratory tract (Hasegawa et al., 2014; Scannapieco, 2021). In pneumonias as well as pleural empyema, both anaerobes and oral bacteria, which can be missed by conventional culture, are more frequent findings by molecular methods (Yamasaki et al., 2013; Dyrhovden et al., 2019; Aoki et al., 2021). In community-acquired pneumonia, approximately $8 \%$ of bronchoalveolar lavage specimens from 64 hospitalized pneumonia patients were positive for Prevotella/Alloprevotella, three with $P$. veroralis, and $P$. melaninogenica and A. tannerae one each (Yamasaki et al., 2013). These findings came from mild cases, however, their pathogenic role remained unknown. Attempts to recover bacterial taxa regardless of their expected pathogenicity or quantity from respiratory specimens revealed Prevotella species in the majority of 17 aspiration pneumonias and eight lung abscesses (Aoki et al., 2021). Only occasional Prevotella recoveries, including P. buccae, P. oris, $P$. pleuritidis, and A. tannerae, came from 27 pleural empyema with poorly described etiology but potentially of oral origin (Dyrhovden et al., 2019).

\section{Prevotella Involvement in Chronic Diseases of the Respiratory Tract}

Chronic diseases of the airways are characterized by a reduced capability of eliminating microbes (Dickson et al., 2016). This may allow a persistent colonization of opportunistic pathogens, such as Pseudomonas aeruginosa or Haemophilus influenzae, with harmful consequences for respiratory health. During exacerbation, there are acute periods resulting in both local and systemic inflammation and worsened lung function. Due to culture-independent methods and increased research interest in the role of anaerobic bacteria and their function, it has been shown that dysbiotic bacterial compositions are, indeed, involved in inflammation of the respiratory airways (Dickson et al., 2016; Huffnagle et al., 2017). The presence of Prevotella in the lower respiratory tract is related to the Th17 activation and 
differentiation, as defined by Th17-chemoattractant chemokine concentrations and STAT3 expression, respectively (Segal et al., 2016). However, the role of Prevotella-mediated Th17 activation in the maintenance of lung health is not fully elucidated yet. A recent mouse-model study demonstrated that the episodic aspiration of oral commensals ( $P$. melaninogenica, $V$. parvula, and S. mitis) leads to dysbiosis and low-dose inflammation in lower airways, decreasing the susceptibility to the respiratory pathogen S. pneumoniae via activation of pulmonary Th17 cells (Wu et al., 2021).

Reduced abundances of the phylum Bacteroidetes and the genus Prevotella have been observed in the oropharynx of patients with asthma and chronic obstructive pulmonary disease (COPD) as well as in bronchial washings in COPD patients (Hilty et al., 2010; Park et al., 2014; Einarsson et al., 2016). A recent study examined pediatric asthma-associated alterations in the respiratory microbiota connected to host metabolism and responses, showing that several Prevotella species were enriched in the control group as well as $P$. pallens and Prevotella oral taxon 306 having an inverse correlation with total and allergen-specific IgE levels (Chiu et al., 2020). In the oropharynx of 13 adult asthma patients, Prevotella proved to be the most abundant genus and $P$. melaninogenica, $P$. pallens, and $P$. nigrescens, in descending order, most abundant Prevotella species (Lopes Dos Santos Santiago et al., 2017). Although P. melaninogenica and S. mitis/S. pneumoniae were the most abundant species, the bacterial compositions did not differ from those found in non-asthmatic individuals. As regards COPD, a recent study characterizing a potential association between the microbiota and risk for exacerbation or airflow limitation revealed significantly reduced proportions of $P$. histicola, Gemella morbillorum, and Streptococcus gordonii in sputum of patients with high risk of COPD exacerbation (Yang et al., 2021). The authors assumed that this kind of alteration in the resident microbiota to a dysbiotic direction could enhance inflammation in respiratory mucosae. In a mouse model, $P$. melaninogenica, $P$. nanceiensis, and $P$. salivae strains were shown to induce COPD-like symptoms via activation of neutrophils and elevating cytokine expression in a TLR-2 dependent manner (Larsen et al., 2014). An interesting finding was that only whole cells but not lipopolysaccharide of Prevotella initiated these symptoms, indicating that TLR-4 does not take part in the cellular response against Prevotella seen in healthy airways of humans. In a previous study, the authors demonstrated that the same Prevotella strains were able to reduce the expression of Haemophilus-induced human dendritic cell IL-12p70, but not IL-23 and IL-10 expressions (Larsen et al., 2012). Later, it was demonstrated that Prevotella outer membrane proteins (OMPs) are responsible for the Th17 development, activation, and IL-17B and IL-17A expression, which eventually promote pulmonary fibrosis (Yang et al., 2019). Noteworthy is that OMPs of Prevotella contain lipopolysaccharides and lipoproteins, which stimulate IL-17 expression via the TLR-Myd88 signaling pathway (Yang et al., 2019). These results indicate that commensal Prevotella organisms not only contribute to the aggravation of airway immune response, but also enable the regulation of pathogeninduced immune response.
Also in other chronic diseases of the lower respiratory tract, like bronchiectasis and cystic fibrosis, Prevotella is among the predominant findings (Tunney et al., 2013; Renwick et al., 2014; Muhlebach et al., 2018; Einarsson et al., 2019). For example, a multi-center study, including over 200 participants with age ranging from childhood to mid-adulthood and with different genetic and geographic backgrounds, examined whether there is a link between strict anaerobes and the severity of cystic fibrosis (Muhlebach et al., 2018). Across all ages, Streptococcus and Prevotella had the highest detection rates in sputum samples, 82 and 51\%, respectively. Contrasting to the high prevalence of Prevotella, its abundance appeared to be low. Interestingly, the presence of anaerobes associated with phenotypically milder disease, whereas Pseudomonas ( $P$. aeruginosa), the typical pathogen in cystic fibrosis, associated with severe disease (Muhlebach et al., 2018). Recently, Einarsson et al. (2019) assessed microbial community structures within the airways and clarified how various taxa are distributed in communities representing health or chronic disease (here: bronchiectasis and cystic fibrosis). The "core" community was composed of members of the genera Streptococcus, Veillonella, Prevotella, Granulicatella, and Fusobacterium, while skewed community structures were found in cystic fibrosis and bronchiectasis samples. Notably, anaerobic bacteria, e.g., Prevotella and Veillonella, proved to affect the variance within the airways, interacting with opportunistic lower airway pathogens (Einarsson et al., 2019).

Some species-level data are available on Prevotella in cystic fibrosis. A recent multi-center study (O'Connor et al., 2021), looking for bacterial communities in bronchoalveolar lavage fluid of 63 diseased and 128 control individuals from infancy to young adulthood, demonstrated the $S$. mitis group (52\%) and $P$. melaninogenica (44\%) as being the most prevalent bacterial taxa. However, distinct abundance patterns were recognized between the study groups; while the abundance of the S. mitis group was high regardless of age in controls, Staphylococcus aureus dominated in cystic fibrosis. Low abundance of $P$. histicola was found in part of diseased and control individuals across the age spectrum, and interestingly, $P$. oris was detected at high abundance in some diseased individuals (O'Connor et al., 2021). Based on the comparison of bronchoalveolar fluid and oropharyngeal swab samples, overall diversity of the upper and lower airway microbiome is similar in clinically stable children with cystic fibrosis (Renwick et al., 2014). However, bacterial communities in lower airways significantly differed between cystic fibrosis and control children; while $P$. veroralis was absent in cystic fibrosis, it was common in controls. Pulsed-field gel electrophoresis patterns were produced in a study targeting to reveal the degree of clonal similarity of 42 Prevotella isolates collected from sputum samples during stable, exacerbated, and post-exacerbation periods (Gilpin et al., 2017). Initial sampling was performed during clinical stability, and the Prevotella findings included $P$. denticola, $P$. histicola, $P$. melaninogenica, $P$. nigrescens, and $P$. salivae. Seven isolates could not be definitely identified but remained as $P$. veroralis $/ P$. histicola or $P$. melaninogenica/P. histicola. Genotyping analysis allowed recognizing similar banding patterns (genotypes) during the 
follow-up. It was suggested that, instead of repeated acquisition, a persistent colonization of Prevotella species had occurred in patients with cystic fibrosis (Gilpin et al., 2017). In an experimental study, cystic fibrosis bronchial epithelial cells were exposed to P. histicola or P. nigrescens (Bertelsen et al., 2021). Both strains were able to induce disrupted NF-кB(p65) and MAPK activations via suppressing TLR-4 and stimulating TLR2 in epithelial cells. Infection with a $P$. nigrescens strain induced only low levels of p65-mediated inflammation compared to inflammatory response of a $P$. aeruginosa strain from the same patient (Bertelsen et al., 2020). The authors speculated that by TLR-2 signaling, and by reducing TLR-4 release and IL- 6 and IL-8 production, Prevotella may inhibit the growth of the major pathogen, $P$. aeruginosa, and have a beneficial effect on immune response in the lungs affected by cystic fibrosis.

\section{SUMMARY}

Due to increased research interest in commensal bacteria in humans, there is now considerable evidence on the complex nature of commensal bacterial communities in the lower airways as well as gastrointestinal tract above the waistline, including the esophagus, stomach, and upper part of the small intestine. It is obvious that oral members of the genus Prevotella play an important role in health and disease at these body sites. In the gastrointestinal tract, the presence of Prevotella may influence, positively or negatively, the severity of disease, such as reflux disease, gastritis, IBD, and different cancer types. In the respiratory tract, current research has brought information on the potential involvement of oral bacteria, including some Prevotella organisms, in COVID-19 persistence and treatment

\section{REFERENCES}

Alauzet, C., Mory, F., Carlier, J. P., Marchandin, H., Jumas-Bilak, E., and Lozniewski, A. (2007). Prevotella nanceiensis sp. nov., isolated from human clinical samples. Int. J. Syst. Evol. Microbiol. 57, 2216-2220. doi: 10.1099/ijs.0. 65173-0

Aoki, K., Ishii, Y., and Tateda, K. (2021). Detection of associated bacteria in aspiration pneumonia and lung abscesses using partial 16S rRNA gene amplicon sequencing. Anaerobe 69:102325. doi: 10.1016/j.anaerobe.2021. 102325

Bassis, C. M., Erb-Downward, J. R., Dickson, R. P., Freeman, C. M., Schmidt, T. M., Young, V. B., et al. (2015). Analysis of the upper respiratory tract microbiotas as the source of the lung and gastric microbiotas in healthy individuals. $m B i o$ 6:e00037. doi: 10.1128/mBio.00037-15

Bertelsen, A., Elborn, J. S., and Schock, B. C. (2020). Infection with Prevotella nigrescens induces TLR2 signalling and low levels of p65 mediated inflammation in Cystic Fibrosis bronchial epithelial cells. J. Cyst. Fibros. 19, 211-218. doi: 10.1016/j.jcf.2019.09.005

Bertelsen, A., Elborn, J. S., and Schock, B. C. (2021). Microbial interaction: Prevotella spp. reduce $P$. aeruginosa induced inflammation in cystic fibrosis bronchial epithelial cells. J. Cyst. Fibros. 20, 682-691. doi: 10.1016/j.jcf.2021. 04.012

Bik, E. M., Eckburg, P. B., Gill, S. R., Nelson, K. E., Purdom, E. A., Francois, F., et al. (2006). Molecular analysis of the bacterial microbiota in the human stomach. Proc. Natl. Acad. Sci. U.S.A. 103, 732-737. doi: 10.1073/pnas.050665 5103

Buhl, M., and Marschal, M. (2020). Prevotella vespertina sp. nov., isolated from an abscess of a hospital patient. Int. J. Syst. Evol. Microbiol. 70, 4576-4582. doi: 10.1099/ijsem.0.004316 outcome. As regards Prevotella species in chronic respiratory diseases, the current data report on reduced abundance of anaerobes, especially Prevotella, which indicates a disruption of homeostatic respiratory microbiota, potentially exposing to lung disease progression. To date, there is only a limited number of mechanistic studies to explain the relation between specific Prevotella species involved in diseases in the respiratory and gastrointestinal tracts. The wide intra-genus variation and distinct properties of individual species within the genus Prevotella call for further studies on oral Prevotella species and their involvement inside and outside the oral cavity to clarify their impact on human health and disease.

\section{AUTHOR CONTRIBUTIONS}

EK conceptualized the manuscript and was responsible for the content dealing with microbes. UKG was responsible for immune-inflammatory aspects. Both authors read and approved the final manuscript.

\section{FUNDING}

A personal grant (EK) of the Finnish Dental Society Apollonia was appreciated.

\section{ACKNOWLEDGMENTS}

We thank Klaus Könönen for generating the anatomical illustration for this article.

Buhl, M., Dunlap, C., and Marschal, M. (2019). Prevotella brunnea sp. nov., isolated from a wound of a patient. Int. J. Syst. Evol. Microbiol. 69, 3933-3938. doi: $10.1099 /$ ijsem. 0.003715

Buhl, M., Willmann, M., Liese, J., Autenrieth, I. B., and Marschal, M. (2016). Prevotella colorans sp. nov., isolated from a human wound. Int. J. Syst. Evol. Microbiol. 66, 3005-3009. doi: 10.1099/ijsem.0.001134

Byrd, K. M., and Gulati, A. S. (2021). The "Gum-Gut" axis in inflammatory bowel diseases: a hypothesis-driven review of associations and advances. Front. Immunol. 12:620124. doi: 10.3389/fimmu.2021.620124

Chiu, C. Y., Chou, H. C., Chang, L. C., Fan, W. L., Dinh, M. C. V., Kuo, Y. L., et al. (2020). Integration of metagenomics-metabolomics reveals specific signatures and functions of airway microbiota in mite-sensitized childhood asthma. Allergy 75, 2846-2857.

Chung, M., Zhao, N., Meier, R., Koestler, D. C., Wu, G., de Castillo, E., et al. (2021). Comparisons of oral, intestinal, and pancreatic bacterial microbiomes in patients with pancreatic cancer and other gastrointestinal diseases. J. Oral Microbiol. 13:1887680. doi: 10.1080/20002297.2021.1887680

Coker, O. O., Dai, Z., Nie, Y., Zhao, G., Cao, L., Nakatsu, G., et al. (2018). Mucosal microbiome dysbiosis in gastric carcinogenesis. Gut 67, 1024-1032. doi: 10.1136/gutjnl-2017-314281

Costello, E. K., Lauber, C. L., Hamady, M., Fierer, N., Gordon, J. I., and Knight, R. (2009). Bacterial community variation in human body habitats across space and time. Science 326, 1694-1697. doi: 10.1126/science.1177486

Das, S., Bernasconi, E., Koutsokera, A., Wurlod, D. A., Tripathi, V., Bonilla-Rosso, G., et al. (2021). A prevalent and culturable microbiota links ecological balance to clinical stability of the human lung after transplantation. Nat. Commun. 12:2126. doi: 10.1038/s41467-021-22344-4

Deshpande, N. P., Riordan, S. M., Castaño-Rodríguez, N., Wilkins, M. R., and Kaakoush, N. O. (2018). Signatures within the esophageal microbiome are 
associated with host genetics, age, and disease. Microbiome 6:227. doi: 10.1186/ s40168-018-0611-4

Dewhirst, F. E., Chen, T., Izard, J., Paster, B. J., Tanner, A. C., Yu, W. H., et al. (2010). The human oral microbiome. J. Bacteriol. 192, 5002-5017. doi: 10.1128/ JB.00542-10

Dickson, R. P., Erb-Downward, J. R., Martinez, F. J., and Huffnagle, G. B. (2016). The microbiome and the respiratory tract. Annu. Rev. Physiol. 78, 481-504. doi: 10.1146/annurev-physiol-021115-105238

Dicksved, J., Lindberg, M., Rosenquist, M., Enroth, H., Jansson, J. K., and Engstrand, L. (2009). Molecular characterization of the stomach microbiota in patients with gastric cancer and in controls. J. Med. Microbiol. 58, 509-516. doi: 10.1099/jmm.0.007302-0

Downes, J., and Wade, W. G. (2011). Prevotella fusca sp. nov. and Prevotella scopos sp. nov., isolated from the human oral cavity. Int. J. Syst. Evol. Microbiol. 61, 854-858. doi: 10.1099/ijs.0.023861-0

Downes, J., Dewhirst, F. E., Tanner, A. C. R., and Wade, W. G. (2013). Description of Alloprevotella rava gen. nov., sp. nov., isolated from the human oral cavity, and reclassification of Prevotella tannerae Moore et al., 1994 as Alloprevotella tannerae gen. nov., comb. nov. Int. J. Syst. Evol. Microbiol. 63, 1214-1218. doi: 10.1099/ijs.0.041376-0

Downes, J., Hooper, S. J., Wilson, M. J., and Wade, W. G. (2008). Prevotella histicola sp. nov., isolated from the human oral cavity. Int. J. Syst. Evol. Microbiol. 58, 1788-1791. doi: 10.1099/ijs.0.65656-0

Downes, J., Liu, M., Kononen, E., and Wade, W. G. (2009). Prevotella micans sp. nov., isolated from the human oral cavity. Int. J. Syst. Evol. Microbiol. 59, 771-774. doi: 10.1099/ijs.0.002337-0

Downes, J., Sutcliffe, I. C., Booth, V., and Wade, W. G. (2007). Prevotella maculosa sp. nov., isolated from the human oral cavity. Int. J. Syst. Evol. Microbiol. 57, 2936-2939. doi: 10.1099/ijs.0.65281-0

Downes, J., Sutcliffe, I. C., Hofstad, T., and Wade, W. G. (2006). Prevotella bergensis sp. nov., isolated from human infections. Int. J. Syst. Evol. Microbiol. 56, 609-612. doi: 10.1099/ijs.0.63888-0

Downes, J., Sutcliffe, I., Tanner, A. C. R., and Wade, W. G. (2005). Prevotella marshii sp. nov. and Prevotella baroniae sp. nov., isolated from the human oral cavity. Int. J. Syst. Evol. Microbiol. 55, 1551-1555. doi: 10.1099/ijs.0.63634-0

Downes, J., Tanner, A. C. R., Dewhirst, F. E., and Wade, W. G. (2010). Prevotella saccharolytica sp. nov., isolated from the human oral cavity. Int. J. Syst. Evol. Microbiol. 60, 2458-2461. doi: 10.1099/ijs.0.014720-0

Dyrhovden, R., Nygaard, R. M., Patel, R., Ulvestad, E., and Kommedal, Ø (2019). The bacterial aetiology of pleural empyema. A descriptive and comparative metagenomic study. Clin. Microbiol. Infect. 25, 981-986. doi: 10.1016/j.cmi. 2018.11.030

Einarsson, G. G., Comer, D. M., McIlreavey, L., Parkhill, J., Ennis, M., Tunney, M. M., et al. (2016). Community dynamics and the lower airway microbiota in stable chronic obstructive pulmonary disease, smokers and healthy nonsmokers. Thorax 71, 795-803. doi: 10.1136/thoraxjnl-2015-207235

Einarsson, G. G., Zhao, J., LiPuma, J. J., Downey, D. G., Tunney, M. M., and Elborn, J. S. (2019). Community analysis and co-occurrence patterns in airway microbial communities during health and disease. ERJ Open Res. 5, 00128-2017. doi: 10.1183/23120541.00128-2017

Finegold, S. M., Strong, C. A., McTeague, M., and Marina, M. (1993). The importance of black-pigmented gram-negative anaerobes in human infections. FEMS Immunol. Med. Microbiol. 6, 77-82. doi: 10.1111/j.1574-695X.1993. tb00306.x

Francavilla, R., Ercolini, D., Piccolo, M., Vannini, L., Siragusa, S., De Filippis, F., et al. (2014). Salivary microbiota and metabolome associated with celiac disease. Appl. Environ. Microbiol. 80, 3416-3425. doi: 10.1128/AEM.00362-14

Gilpin, D. F., Nixon, K. A., Bull, M., McGrath, S. J., Sherrard, L., Rolain, J. M., et al. (2017). Evidence of persistence of Prevotella spp. in the cystic fibrosis lung. J. Med. Microbiol. 66, 825-832. doi: 10.1099/jmm.0.000500

Glazunova, O. O., Launay, T., Raoult, D., and Roux, V. (2007). Prevotella timonensis sp. nov., isolated from a human breast abscess. Int. J. Syst. Evol. Microbiol. 57, 883-886. doi: 10.1099/ijs.0.64609-0

Han, H. S., Lee, S. Y., Oh, S. Y., Moon, H. W., Cho, H., and Kim, J. H. (2019). Correlations of the gastric and duodenal microbiota with histological, endoscopic, and symptomatic gastritis. J. Clin. Med. 8:312. doi: 10.3390/ jcm8030312
Haran, J. P., Bradley, E., Zeamer, A. L., Cincotta, L., Salive, M. C., Dutta, P., et al. (2021). Inflammation-type dysbiosis of the oral microbiome associates with the duration of COVID-19 symptoms and long-COVID. JCI Insight 17:152346. doi: $10.1172 /$ jci.insight.152346

Hasegawa, A., Sato, T., Hoshikawa, Y., Ishida, N., Tanda, N., Kawamura, Y., et al. (2014). Detection and identification of oral anaerobes in intraoperative bronchial fluids of patients with pulmonary carcinoma. Microbiol. Immunol. 58, 375-381. doi: 10.1111/1348-0421.12157

Hedberg, M. E., Israelsson, A., Moore, E. R. B., Svensson-Stadler, L., Wai, S. N., Pietz, G., et al. (2013). Prevotella jejuni sp. nov., isolated from the small intestine of a child with coeliac disease. Int. J. Syst. Evol. Microbiol. 63, 4218-4223. doi: 10.1099/ijs.0.052647-0

Hilty, M., Burke, C., Pedro, H., Cardenas, P., Bush, A., Bossley, C., et al. (2010). Disordered microbial communities in asthmatic airways. PLoS One 5:e8578. doi: 10.1371/journal.pone.0008578

Huang, J., Roosaar, A., Axéll, T., and Ye, W. (2016). A prospective cohort study on poor oral hygiene and pancreatic cancer risk. Int. J. Cancer 138, 340-347. doi: $10.1002 /$ ijc. 29710

Huffnagle, G. B., Dickson, R. P., and Lukacs, N. W. (2017). The respiratory tract microbiome and lung inflammation: a two-way street. Mucosal. Immunol. 10, 299-306. doi: 10.1038/mi.2016.108

Humphrey, S. P., and Williamson, R. T. (2001). A review of saliva: normal composition, flow, and function. J. Prosthet. Dent. 85, 162-169. doi: 10.1067/ mpr.2001.113778

Jensen, A., Fagö-Olsen, H., Sørensen, C. H., and Kilian, M. (2013). Molecular mapping to species level of the tonsillar crypt microbiota associated with health and recurrent tonsillitis. PLoS One 8:e56418. doi: 10.1371/journal.pone. 0056418

Jousimies-Somer, H., Summanen, P., Citron, D. M., Baron, E. J., Wexler, H. M., and Finegold, S. M. (2002). Wadsworth-KTL Anaerobic Bacteriology Manual. Belmont, CA: Star Publishing.

Kawar, N., Park, S. G., Schwartz, J. L., Callahan, N., Obrez, A., Yang, B., et al. (2021). Salivary microbiome with gastroesophageal reflux disease and treatment. Sci. Rep. 11:188. doi: 10.1038/s41598-020-80170-y

Keijser, B. J., Zaura, E., Huse, S. M., van der Vossen, J. M., Schuren, F. H., Montijn, R. C., et al. (2008). Pyrosequencing analysis of the oral microflora of healthy adults. J. Dent. Res. 87, 1016-1020. doi: 10.1177/154405910808701104

Kitamoto, S., Nagao-Kitamoto, H., Hein, R., Schmidt, T. M., and Kamada, N. (2020). The bacterial connection between the oral cavity and the gut diseases. J. Dent. Res. 99, 1021-1029. doi: 10.1177/0022034520924633

Könönen, E. (2005). Anaerobes in the upper respiratory tract in infancy. Anaerobe 11, 131-136. doi: 10.1016/j.anaerobe.2004.11.001

Könönen, E., Eerola, E., Frandsen, E. V., Jalava, J., Mättö, J., Salmenlinna, S., et al. (1998). Phylogenetic characterization and proposal of a new pigmented species to the genus Prevotella: Prevotella pallens sp. nov. Int. J. Syst. Bacteriol. 48, 47-51. doi: 10.1099/00207713-48-1-47

Könönen, E., Kanervo, A., Takala, A., Asikainen, S., and Jousimies-Somer, H. (1999). Establishment of oral anaerobes during the first year of life. J. Dent. Res. 78, 1634-1639. doi: 10.1177/00220345990780100801

Larsen, J. M., Musavian, H. S., Butt, T. M., Ingvorsen, C., Thysen, A. H., and Brix, S. (2014). Chronic obstructive pulmonary disease and asthma-associated Proteobacteria, but not commensal Prevotella spp., promote Toll-like receptor 2-independent lung inflammation and pathology. Immunology 144, 333-342. doi: $10.1111 /$ imm. 12376

Larsen, J. M., Steen-Jensen, D. B., Laursen, J. M., Søndergaard, J. N., Musavian, H. S., Butt, T. M., et al. (2012). Divergent pro-inflammatory profile of human dendritic cells in response to commensal and pathogenic bacteria associated with the airway microbiota. PLoS One 7:e31976.

Lawson, P. A., Moore, E., and Falsen, E. (2008). Prevotella amnii sp. nov., isolated from human amniotic fluid. Int. J. Syst. Evol. Microbiol. 58, 89-92. doi: 10.1099/ ijs.0.65118-0

Ley, R. E. (2016). Gut microbiota in 2015: Prevotella in the gut: choose carefully. Nat. Rev. Gastroenterol. Hepatol. 13, 69-70. doi: 10.1038/nrgastro.2016.4

Liu, N., Ando, T., Ishiguro, K., Maeda, O., Watanabe, O., Funasaka, K., et al. (2013). Characterization of bacterial biota in the distal esophagus of Japanese patients with reflux esophagitis and Barrett's esophagus. BMC Infect. Dis. 13:130. doi: 10.1186/1471-2334-13-130 
Liu, Y., Lin, Z., Lin, Y., Chen, Y., Peng, X. E., He, F., et al. (2018). Streptococcus and Prevotella are associated with the prognosis of oesophageal squamous cell carcinoma. J. Med. Microbiol. 67, 1058-1068. doi: 10.1099/jmm.0.000754

Lopes Dos Santos Santiago, G., Brusselle, G., Dauwe, K., Deschaght, P., Verhofstede, C., Vaneechoutte, D., et al. (2017). Influence of chronic azithromycin treatment on the composition of the oropharyngeal microbial community in patients with severe asthma. BMC Microbiol. 17:109. doi: 10 . 1186/s12866-017-1022-6

Lopetuso, L. R., Severgnini, M., Pecere, S., Ponziani, F. R., Boskoski, I., Larghi, A., et al. (2020). Esophageal microbiome signature in patients with Barrett's esophagus and esophageal adenocarcinoma. PLoS One 15:e0231789. doi: 10. 1371/journal.pone.0231789

Mager, D. L., Ximenez-Fyvie, L. A., Haffajee, A. D., and Socransky, S. S. (2003). Distribution of selected bacterial species on intraoral surfaces. J. Clin. Periodontol. 30, 644-654. doi: 10.1034/j.1600-051x.2003.00376.x

Mentula, S., Harmoinen, J., Heikkilä, M., Westermarck, E., Rautio, M., Huovinen, P., et al. (2005). Comparison between cultured small-intestinal and fecal microbiotas in beagle dogs. Appl. Environ. Microbiol. 71, 4169-4175. doi: 10. 1128/AEM.71.8.4169-4175.2005

Miller, E. H., Annavajhala, M. K., Chong, A. M., Park, H., Nobel, Y. R., Soroush, A., et al. (2021). Oral microbiome alterations and SARS-CoV-2 saliva viral load in patients with COVID-19. Microbiol. Spectr. 13:e0005521. doi: 10.1128/ Spectrum.00055-21

Moore, L. V., Johnson, J. L., and Moore, W. E. (1994). Descriptions of Prevotella tannerae sp. nov. and Prevotella enoeca sp. nov. from the human gingival crevice and emendation of the description of Prevotella zoogleoformans. Int. J. Syst. Bacteriol. 44, 599-602. doi: 10.1099/00207713-44-4-599

Muhlebach, M. S., Hatch, J. E., Einarsson, G. G., McGrath, S. J., Gilipin, D. F., Lavelle, G., et al. (2018). Anaerobic bacteria cultured from cystic fibrosis airways correlate to milder disease: a multisite study. Eur. Respir. J. 52:1800242. doi: 10.1183/13993003.00242-2018

O’Connor, J. B., Mottlowitz, M. M., Wagner, B. D., Boyne, K. L., Stevens, M. J., Robertson, C. E., et al. (2021). Divergence of bacterial communities in the lower airways of CF patients in early childhood. PLoS One 16:e257838. doi: 10.1371/journal.pone.0257838

Park, H., Shin, J. W., Park, S. G., and Kim, W. (2014). Microbial communities in the upper respiratory tract of patients with asthma and chronic obstructive pulmonary disease. PLoS One 9:e109710. doi: 10.1371/journal.pone.0109710

Pattaroni, C., Watzenboeck, M. L., Schneidegger, S., Kieser, S., Wong, N. C., Bernasconi, E., et al. (2018). Early-life formation of the microbial and immunological environment of the human airways. Cell Host Microbe 24, 857-865.e4. doi: 10.1016/j.chom.2018.10.019

Pei, Z., Bini, E. J., Yang, L., Zhou, M., Francois, F., and Blaser, M. J. (2004). Bacterial biota in the human distal esophagus. Proc. Natl. Acad. Sci. U.S.A. 101, 4250-4255. doi: 10.1073/pnas.0306398101

Qi, Y., Zang, S. Q., Wei, J., Yu, H. C., Yang, Z., Wu, H. M., et al. (2021). High-throughput sequencing provides insights into oral microbiota dysbiosis in association with inflammatory bowel disease. Genomics 113, 664-676. doi: 10.1016/j.ygeno.2020.09.063

Renwick, J., McNally, P., John, B., DeSantis, T., Linnane, B., Murphy, P., et al. (2014). The microbial community of the cystic fibrosis airway is disrupted in early life. PLoS One 9:e109798. doi: 10.1371/journal.pone.0109798

Said, H. S., Suda, W., Nakagome, S., Chinen, H., Oshima, K., Kim, S., et al. (2014). Dysbiosis of salivary microbiota in inflammatory bowel disease and its association with oral immunological biomarkers. DNA Res. 21, 15-25. doi: 10.1093/dnares/dst037

Sakamoto, M., Huang, Y., Umeda, M., Ishikawa, I., and Benno, Y. (2005a). Prevotella multiformis sp. nov., isolated from human subgingival plaque. Int. J. Syst. Evol. Microbiol. 55, 815-819. doi: 10.1099/ijs.0.63451-0

Sakamoto, M., Ohkusu, K., Masaki, T., Kako, H., Ezaki, T., and Benno, Y. (2007). Prevotella pleuritidis sp. nov., isolated from pleural fluid. Int. J. Syst. Evol. Microbiol. 57, 1725-1728. doi: 10.1099/ijs.0.64885-0

Sakamoto, M., Suzuki, M., Huang, Y., Umeda, M., Ishikawa, I., and Benno, Y. (2004). Prevotella shahii sp. nov. and Prevotella salivae sp. nov., isolated from the human oral cavity. Int. J. Syst. Evol. Microbiol. 54, 877-883. doi: 10.1099/ijs. 0.02876-0

Sakamoto, M., Suzuki, N., and Okamoto, M. (2010). Prevotella aurantiaca sp. nov., isolated from the human oral cavity. Int. J. Syst. Evol. Microbiol. 60, 500-503. doi: 10.1099/ijs.0.012831-0
Sakamoto, M., Umeda, M., Ishikawa, I., and Benno, Y. (2005b). Prevotella multisaccharivorax sp. nov., isolated from human subgingival plaque. Int. J. Syst. Evol. Microbiol. 55, 1839-1843. doi: 10.1099/ijs.0.63739-0

Scannapieco, F. A. (2021). Poor oral health in the etiology and prevention of aspiration pneumonia. Dent. Clin. North Am. 65, 307-321. doi: 10.1016/j.cden. 2020.11.006

Schmidt, T. S., Hayward, M. R., Coelho, L. P., Li, S. S., Costea, P. I., Voigt, A. Y., et al. (2019). Extensive transmission of microbes along the gastrointestinal tract. eLife 8:e42693. doi: 10.7554/eLife.42693

Schulz, C., Schütte, K., Koch, N., Vilchez-Vargas, R., Wos-Oxley, M. L., Oxley, A. P. A., et al. (2018). The active bacterial assemblages of the upper GI tract in individuals with and without Helicobacter infection. Gut 67, 216-225. doi: 10.1136/gutjnl-2016-312904

Segal, L. N., Clemente, J. C., Tsay, J. C., Koralov, S. B., Keller, B. C., Wu, B. G., et al. (2016). Enrichment of the lung microbiome with oral taxa is associated with lung inflammation of a Th17 phenotype. Nat. Microbiol. 1:16031. doi: 10.1038/nmicrobiol.2016.31

Segata, N., Haake, S. K., Mannon, P., Lemon, K. P., Waldron, L., Gevers, D., et al. (2012). Composition of the adult digestive tract bacterial microbiome based on seven mouth surfaces, tonsils, throat and stool samples. Genome Biol. 13:R42. doi: 10.1186/gb-2012-13-6-r42

Shah, H. N., and Collins, D. M. (1990). Prevotella, a new genus to include Bacteroides melaninogenicus and related species formerly classified in the genus Bacteroides. Int. J. Syst. Bacteriol. 40, 205-208. doi: 10.1099/00207713-40-2-205

Shah, H. N., and Gharbia, S. E. (1992). Biochemical and chemical studies on strains designated Prevotella intermedia and proposal of a new pigmented species, Prevotella nigrescens sp. nov. Int. J. Syst. Bacteriol. 42, 542-546. doi: 10.1099/ 00207713-42-4-542

Shanahan, E. R., Shah, A., Koloski, N., Walker, M. M., Talley, N. J., Morrison, M., et al. (2018). Influence of cigarette smoking on the human duodenal mucosa-associated microbiota. Microbiome 6:150. doi: 10.1186/s40168-0180531-3

Sulaiman, I., Chung, M., Angel, L., Tsay, J. J., Wu, B. G., Yeung, S. T., et al. (2021). Microbial signatures in the lower airways of mechanically ventilated COVID19 patients associated with poor clinical outcome. Nat. Microbiol. 6, 1245-1258. doi: 10.1038/s41564-021-00961-5

Sung, H., Ferlay, J., Siegel, R. L., Laversanne, M., Soerjomataram, I., Jemal, A., et al. (2021). Global Cancer Statistics 2020: GLOBOCAN estimates of incidence and mortality worldwide for 36 cancers in 185 countries. CA Cancer J. Clin. 71, 209-249. doi: 10.3322/caac.21660

Tett, A., Pasolli, E., Masetti, G., Ercolini, D., and Segata, N. (2021). Prevotella diversity, niches and interactions with the human host. Nat. Rev. Microbiol. 19, 585-599. doi: 10.1038/s41579-021-00559-y

Tunney, M. M., Einarsson, G. G., Wei, L., Drain, M., Klem, E. R., Cardwell, C., et al. (2013). Lung microbiota and bacterial abundance in patients with bronchiectasis when clinically stable and during exacerbation. Am. J. Respir. Crit. Care Med. 187, 1118-1126. doi: 10.1164/rccm.201210-1937OC

Vasapolli, R., Schütte, K., Schulz, C., Vital, M., Schomburg, D., Pieper, D. H., et al. (2019). Analysis of transcriptionally active bacteria throughout the gastrointestinal tract of healthy individuals. Gastroenterology 157, 10811092.e3. doi: 10.1053/j.gastro.2019.05.068

Willems, A., and Collins, M. D. (1995). 16S rRNA gene similarities indicate that Hallella seregens (Moore and Moore) and Mitsuokella dentalis (Haapsalo et al.) are genealogically highly related and are members of the genus Prevotella: emended description of the genus Prevotella (Shah and Collins) and description of Prevotella dentalis comb. nov. Int. J. Syst. Bacteriol. 45, 832-836. doi: 10.1099/ 00207713-45-4-832

Wu, B. G., Sulaiman, I., Tsay, J. J., Perez, L., Franca, B., Li, Y., et al. (2021). Episodic aspiration with oral commensals induces a MyD88-dependent, pulmonary T-helper cell type 17 response that mitigates susceptibility to Streptococcus pneumoniae. Am. J. Respir. Crit. Care Med. 203, 1099-1111. doi: 10.1164/rccm. 202005-1596OC

Xiong, D., Muema, C., Zhang, X., Pan, X., Xiong, J., Yang, H., et al. (2021). Enriched opportunistic pathogens revealed by metagenomic sequencing hint potential linkages between pharyngeal microbiota and COVID-19. Virol. Sin. 12, 1-10. doi: 10.1007/s12250-021-00391-X

Xu, X., He, J., Xue, J., Wang, Y., Li, K., Zhang, K., et al. (2015). Oral cavity contains distinct niches with dynamic microbial communities. Environ. Microbiol. 17, 699-710. doi: 10.1111/1462-2920.12502 
Xun, Z., Zhang, Q., Xu, T., Chen, N., and Chen, F. (2018). Dysbiosis and ecotypes of the salivary microbiome associated with inflammatory bowel diseases and the assistance in diagnosis of diseases using oral bacterial profiles. Front. Microbiol. 9:1136. doi: 10.3389/fmicb.2018.01136

Yamasaki, K., Kawanami, T., Yatera, K., Fukuda, K., Noguchi, S., Nagata, S., et al. (2013). Significance of anaerobes and oral bacteria in community-acquired pneumonia. PLoS One 8:e63103. doi: 10.1371/journal.pone.0063103

Yang, C. Y., Li, S. W., Chin, C. Y., Hsu, C. W., Lee, C. C., Yeh, Y. M., et al. (2021). Association of exacerbation phenotype with the sputum microbiome in chronic obstructive pulmonary disease patients during the clinically stable state. J. Transl. Med. 19:121. doi: 10.1186/s12967-021-02 788-4

Yang, D., Chen, X., Wang, J., Lou, Q., Lou, Y., Li, L., et al. (2019). Dysregulated lung commensal bacteria drive interleukin-17b production to promote pulmonary fibrosis through their outer membrane vesicles. Immunity 19, 692-706. doi: 10.1016/j.immuni.2019.02.001
Conflict of Interest: The authors declare that the research was conducted in the absence of any commercial or financial relationships that could be construed as a potential conflict of interest.

Publisher's Note: All claims expressed in this article are solely those of the authors and do not necessarily represent those of their affiliated organizations, or those of the publisher, the editors and the reviewers. Any product that may be evaluated in this article, or claim that may be made by its manufacturer, is not guaranteed or endorsed by the publisher.

Copyright (C) 2022 Könönen and Gursoy. This is an open-access article distributed under the terms of the Creative Commons Attribution License (CC BY). The use, distribution or reproduction in other forums is permitted, provided the original author(s) and the copyright owner(s) are credited and that the original publication in this journal is cited, in accordance with accepted academic practice. No use, distribution or reproduction is permitted which does not comply with these terms. 